\title{
Genome-wide sequencing as a first-tier screening test for short tandem repeat expansions
}

Indhu-Shree Rajan-Babu ${ }^{1,2^{*}}$, Junran J. Peng ${ }^{1}$, Readman Chiư ${ }^{3}$, IMAGINE Study ${ }^{1}$, CAUSES Study ${ }^{1}$, Chenkai Li $i^{3,4}$, Arezoo Mohajeri ${ }^{1}$, Egor Dolzhenko ${ }^{5}$, Michael A. Eberle ${ }^{5}$, Inanc Birol ${ }^{1,3}$ and Jan M. Friedman ${ }^{1}$

\begin{abstract}
Background: Screening for short tandem repeat (STR) expansions in next-generation sequencing data can enable diagnosis, optimal clinical management/treatment, and accurate genetic counseling of patients with repeat expansion disorders. We aimed to develop an efficient computational workflow for reliable detection of STR expansions in next-generation sequencing data and demonstrate its clinical utility.

Methods: We characterized the performance of eight STR analysis methods (lobSTR, HipSTR, RepeatSeq, ExpansionHunter, TREDPARSE, GangSTR, STRetch, and exSTRa) on next-generation sequencing datasets of samples with known disease-causing full-mutation STR expansions and genomes simulated to harbor repeat expansions at selected loci and optimized their sensitivity. We then used a machine learning decision tree classifier to identify an optimal combination of methods for full-mutation detection. In Burrows-Wheeler Aligner (BWA)-aligned genomes, the ensemble approach of using ExpansionHunter, STRetch, and exSTRa performed the best (precision $=82 \%$, recall $=100 \%$, F1-score $=90 \%)$. We applied this pipeline to screen 301 families of children with suspected genetic disorders.

Results: We identified 10 individuals with full-mutations in the AR, ATXN1, ATXN8, DMPK, FXN, or HTT disease STR locus in the analyzed families. Additional candidates identified in our analysis include two probands with borderline ATXN2 expansions between the established repeat size range for reduced-penetrance and full-penetrance fullmutation and seven individuals with FMR1 CGG repeats in the intermediate/premutation repeat size range. In 67 probands with a prior negative clinical PCR test for the FMR1, FXN, or DMPK disease STR locus, or the spinocerebellar ataxia disease STR panel, our pipeline did not falsely identify aberrant expansion. We performed clinical PCR tests on seven (out of 10) full-mutation samples identified by our pipeline and confirmed the expansion status in all, showing absolute concordance between our bioinformatics and molecular findings.
\end{abstract}

\footnotetext{
* Correspondence: indhu.babu@bcchr.ca

'Department of Medical Genetics, University of British Columbia and Children's \& Women's Hospital, Vancouver, BC V6H3N1, Canada

2Department of Medical and Molecular Genetics, King's College London, Strand, London WC2R 2LS, UK

Full list of author information is available at the end of the article
}

(c) The Author(s). 2021, corrected publication 2021. Open Access This article is licensed under a Creative Commons Attribution 4.0 International License, which permits use, sharing, adaptation, distribution and reproduction in any medium or format, as long as you give appropriate credit to the original author(s) and the source, provide a link to the Creative Commons licence, and indicate if changes were made. The images or other third party material in this article are included in the article's Creative Commons licence, unless indicated otherwise in a credit line to the material. If material is not included in the article's Creative Commons licence and your intended use is not permitted by statutory regulation or exceeds the permitted use, you will need to obtain permission directly from the copyright holder. To view a copy of this licence, visit http://creativecommons.org/ licenses/by/4.0/. The Creative Commons Public Domain Dedication waiver (http://creativecommons.org/publicdomain/zero/1. 0/) applies to the data made available in this article, unless otherwise stated in a credit line to the data. 
Conclusions: We have successfully demonstrated the application of a well-optimized bioinformatics pipeline that promotes the utility of genome-wide sequencing as a first-tier screening test to detect expansions of known disease STRs. Interrogating clinical next-generation sequencing data for pathogenic STR expansions using our ensemble pipeline can improve diagnostic yield and enhance clinical outcomes for patients with repeat expansion disorders.

Keywords: Clinical bioinformatics, Repeat expansion, Next-generation sequencing, Short tandem repeats, Machine learning

\section{Background}

Expansions of short tandem repeats (STRs; tandemly repeated arrays of 1-6 base pair (bp) sequence motifs [1]) can cause several neurological and neuromuscular disorders [2]. Accurate genotyping (i.e., the determination of the number of copies of repeat units in an STR) is critical to the molecular diagnosis of STR expansion disorders as repeat length often influences disease severity and age of onset of clinical symptoms [3]. Repeat length also determines an STR's allelic class (normal, intermediate, premutation, or full-mutation), which may differ with respect to the associated disease phenotype [3, 4]. For example, the FMR1 (MIM 309550) premutation (55-200 CGG repeats) increases the risk for primary ovarian insufficiency (MIM 311360) and tremor/ataxia syndrome (MIM 300623). In contrast, FMR1 fullmutation (> 200 CGG repeats) causes fragile $\mathrm{X}$ syndrome (MIM 300624), the most frequent Mendelian cause of intellectual disability [5]. Premutation and intermediate (also known as "mutable normal") alleles that are meiotically unstable can expand into pathogenic fullmutation in a single generation, while normal alleles rarely, if ever, do so [6, 7]. Expanded alleles tend to further increase in repeat length during intergenerational transmission, and as a result, genetic anticipation (the earlier and more severe manifestation of disease symptoms with each successive generation) is common in repeat expansion disorders [8].

Clinical laboratories typically use polymerase chain reaction (PCR) or Southern blot (alone or in combination) to characterize expansions at known disease STR loci [9]. Although highly sensitive in detecting and genotyping STR expansions, PCR and Southern blot tests have several limitations. They are time- and labor-intensive, require extensive optimization, and do not permit concurrent analyses of more than a handful of STR loci. Next-generation sequencing (NGS), on the other hand, enables exome- or genome-wide characterization of STRs.

Several algorithms have recently been developed to analyze STRs in NGS data [1, 10-14]. The incorporation of bioinformatics tools to screen for STR expansions may permit the diagnosis of repeat expansion disorders during routine diagnostic exome or genome sequencing, allowing accurate genetic counseling of affected individuals and their families, and improving clinical outcomes. The currently available STR analysis algorithms have different attributes that determine their utility and sensitivity in detecting and characterizing repeat expansions in NGS data (Table 1). Methods like STRetch [11] and exSTRa [12] identify STR expansions via case-control analysis, with a caveat of either underestimating the repeat lengths of some expanded STRs [11] or not providing repeat length estimates at all [12]. Methods that genotype STRs perform better across certain repeat length ranges depending on the read type evidence considered. For instance, lobSTR [15], HipSTR [16], and RepeatSeq [17], which only rely on reads that fully encompass an STR ("spanning reads") to compute repeat length, can size alleles within the length of an Illumina read (125-150 bp) but perform poorly in detecting pathogenic full-mutation expansions that exceed read length. More recent methods $[1,10,13,14]$ that leverage additional read types such as flanking or partially flanking reads (those that map to unique flanking sequences), in-repeat reads (IRR; those that are entirely composed of STRs with a read-pair mate that maps to the STR's flanking sequence), and/or IRR pairs (both reads of a pair mapping to the STR) can size STRs that exceed read length. ExpansionHunter $(E H)[10$, 13] and GangSTR [14], in particular, enable the recovery of IRR and IRR pairs, which originate from an expanded STR but may incorrectly map to other STR (or "off-target") regions with longer tracts of the same repeat motif. By allowing the inclusion of off-target sites (OTS), EH and GangSTR analyses facilitate sizing STRs that are longer than an Illumina sequencing library fragment length (350-500 bp).

In terms of utility, some of these methods can analyze STRs in both exome sequencing (ES) and whole-genome sequencing (WGS) data [11, 12, 14], while others are designed specifically for WGS $[1,10,13]$. Some tools have specific NGS data requirements; for example, EH is designed for PCR-free WGS, and exSTRa has only been extensively tested on bowtie 2 [18] alignments. Also, most methods perform less well on GC-rich STR expansions $[10,12]$.

These varied attributes and performance characteristics have led to the acknowledgement that a single 
Table 1 Features of some publicly available short tandem repeat analysis algorithms

\begin{tabular}{|c|c|c|c|c|c|c|c|c|}
\hline Features & lobSTR & RepeatSeq & HipSTR & TRED & EH & STRetch & exSTRa & GangSTR \\
\hline $\begin{array}{l}\text { Outputs } \\
\text { repeat length? }\end{array}$ & $Y$ & $Y$ & $Y$ & $Y$ & $Y$ & $Y$ & $N$ & $Y$ \\
\hline $\begin{array}{l}\text { Sequencing } \\
\text { reads }\end{array}$ & $\begin{array}{l}\text { Single- and } \\
\text { paired-end }\end{array}$ & $\begin{array}{l}\text { Single- and } \\
\text { paired-end }\end{array}$ & $\begin{array}{l}\text { Single- } \\
\text { and } \\
\text { paired- } \\
\text { end }\end{array}$ & Paired-end & Paired-end & $\begin{array}{l}\text { Paired- } \\
\text { end }\end{array}$ & Paired-end & Paired-end \\
\hline $\begin{array}{l}\text { Sequencing } \\
\text { platforms } \\
\text { supported }\end{array}$ & $\begin{array}{l}\text { Illumina, Sanger, } \\
454 \text {, and } \\
\text { lonTorrent }\end{array}$ & Illumina & Illumina & Illumina & Illumina & Illumina & Illumina & Illumina \\
\hline $\begin{array}{l}\text { Library prep. } \\
\text { supported }\end{array}$ & $\begin{array}{l}P C R \text { and PCR- } \\
\text { free }\end{array}$ & n.a. & $\begin{array}{l}\text { PCR and } \\
\text { PCR-free }\end{array}$ & $P C R$ and $P C R$-free & $\begin{array}{l}P C R \text { and PCR- } \\
\text { free }\end{array}$ & $\begin{array}{l}\text { PCR and } \\
\text { PCR-free }\end{array}$ & $\begin{array}{l}P C R \text { and PCR- } \\
\text { free }\end{array}$ & $\begin{array}{l}\text { PCR and PCR- } \\
\text { free }\end{array}$ \\
\hline $\begin{array}{l}\text { Library prep. } \\
\text { (rcmd) }\end{array}$ & None & None & None & None & PCR-free & PCR-free & None & None \\
\hline $\begin{array}{l}\text { Aligners } \\
\text { (rcmd) }\end{array}$ & $\begin{array}{l}\text { lobSTR and } \\
\text { BWA-MEM }\end{array}$ & $\begin{array}{l}\text { Novoalign } \\
\text { and Bowtie } \\
2\end{array}$ & $\begin{array}{l}\text { Indel- } \\
\text { sensitive } \\
\text { aligner }\end{array}$ & None & None & None & Bowtie 2 & None \\
\hline $\begin{array}{l}\text { Analysis } \\
\text { approach }\end{array}$ & $\begin{array}{l}\text { Targeted and } \\
\text { GW }\end{array}$ & $\begin{array}{l}\text { Targeted } \\
\text { and GW }\end{array}$ & $\begin{array}{l}\text { Targeted } \\
\text { and GW }\end{array}$ & Targeted & Targeted & GW & $\begin{array}{l}\text { Targeted and } \\
\text { GW }\end{array}$ & $\begin{array}{l}\text { Targeted and } \\
\text { GW }\end{array}$ \\
\hline $\begin{array}{l}\text { NGS data type } \\
\text { supported }\end{array}$ & WGS & WGS & WGS & WGS & WGS and ES & $\begin{array}{l}\text { WGS and } \\
\text { ES }\end{array}$ & WGS and ES & WGS and ES \\
\hline $\begin{array}{l}\text { NGS data } \\
\text { format }\end{array}$ & $\begin{array}{l}\text { bam, fastq, or } \\
\text { fasta }\end{array}$ & bam & bam & bam & bam or .cram & $\begin{array}{l}\text { bam or } \\
\text { fastq }\end{array}$ & bam & bam \\
\hline $\begin{array}{l}\text { Built-in stutter } \\
\text { correction } \\
\text { model }\end{array}$ & Y & Y & Y & Y & n.a. & n.a. & n.a. & Y \\
\hline $\begin{array}{l}\text { Test of } \\
\text { significance }\end{array}$ & N & N & N & N & $N$ & Y & Y & N \\
\hline $\begin{array}{l}\text { Read types } \\
\text { used }\end{array}$ & Spanning & Spanning & Spanning & $\begin{array}{l}\text { Spanning, flanking or } \\
\text { partial, paired-end reads, } \\
\text { and IRR }\end{array}$ & $\begin{array}{l}\text { Spanning, } \\
\text { flanking, and } \\
\text { IRR/IRR pairs }\end{array}$ & $\begin{array}{l}\text { Anchored } \\
\text { IRR }\end{array}$ & $\begin{array}{l}\text { Flanking and } \\
\text { anchored IRR }\end{array}$ & $\begin{array}{l}\text { Spanning, } \\
\text { flanking, and } \\
\text { IRR/IRR pairs }\end{array}$ \\
\hline Phasing $^{\mathrm{b}}$ & n.a & n.a & Y & n.a & n.a & n.a & n.a & n.a \\
\hline$P L$ & $\mathrm{C}++$ & $\mathrm{C}++$ & $\mathrm{C}++$ & Python & $\mathrm{C}++$ & Java & Perl and $\mathrm{R}$ & $\mathrm{C}++$ \\
\hline $\begin{array}{l}\text { Sizing } \\
\text { limitation }\end{array}$ & $\mathrm{RL}$ & $\mathrm{RL}$ & $\mathrm{RL}$ & $\mathrm{FL}$ & Not limited & $\mathrm{FL}$ & n.a. & Not limited \\
\hline $\begin{array}{l}\text { Control } \\
\text { dataset }\end{array}$ & Not required & $\begin{array}{l}\text { Not } \\
\text { required }\end{array}$ & $\begin{array}{l}\text { Not } \\
\text { required }\end{array}$ & Not required & Not required & Required & Not required & Not required \\
\hline $\begin{array}{l}\text { Complex } \\
\text { repeats }\end{array}$ & n.a. & n.a. & n.a. & n.a. & Y & n.a. & n.a. & N \\
\hline Output files & $\begin{array}{l}. v c f \text { and } \\
\text {.allelotype.stats }\end{array}$ & $\begin{array}{l}\text { repeatseq, } \\
\text {.calls, and } \\
. v c f\end{array}$ &.$v c f$ & .vcf and .json & $\begin{array}{l}. v c f, \text { json, and } \\
. \log \end{array}$ &.$t s v$ & $\begin{array}{l}\text { p-values, } \\
\text { ECDF, and } \\
\text { tsum plots }\end{array}$ &.$v c f$ \\
\hline $\begin{array}{l}\text { Customized } \\
\text { regions file }\end{array}$ & Possible & Possible & Possible & Possible & Possible & $\begin{array}{l}\text { Possible, } \\
\text { but not } \\
\text { remd }\end{array}$ & Possible & Possible \\
\hline
\end{tabular}

EH ExpansionHunter, TRED TREDPARSE, $Y$ feature included, $N$ feature not included, Library prep library preparation protocol, $r c m d$ recommended, $P L$ programming language used, n.a. not applicable/information not available, GW genome-wide, WGS whole-genome sequencing, ES exome sequencing, IRR in-repeat reads, $R L$ read length, $F L$ fragment length, Not limited not limited by either RL or FL, ECDF Empirical Cumulative Distribution Function, $t$-sum aggregated $T$ statistic ${ }^{a}$ Corrects the noise (stutters) introduced during PCR amplification-based library preparation

butilizes phased single nucleotide variant haplotypes

bioinformatics tool is less likely to be able to identify pathogenic STR expansions of all repeat lengths and sequence content/composition in NGS data [12]. Recently, Tankard et al. recommended a consensus calling approach using at least two out of four tools (TREDPARSE [1], EH, STRetch, and exSTRa) to characterize expansions of known disease STRs [12]. However, it is not clear which of these (or other) STR methods alone or in combination yield optimal sensitivity and specificity.

In this study, we employed a decision tree classifier to identify the optimal tool(s) for classifying expanded full- 
mutation and non-expanded alleles at known disease STR loci with high accuracy, precision, recall, and F1score. We performed our analysis on STR calls from eight different tools (lobSTR, HipSTR, RepeatSeq, EH, TREDPARSE, GangSTR, STRetch, and exSTRa) made on WGS data of patients with well-characterized STR expansions in one of eight different loci ( $A R, A T N 1$, ATXN1, ATXN3, DMPK, FMR1, FXN, or HTT) and simulated WGS data harboring expansions at GC-rich FMR1, FMR2, or C9orf72 STR loci. We included the spanning-read-only algorithms (lobSTR, HipSTR, and RepeatSeq), which have been used in recent studies to characterize normal polymorphic STR variations [19, 20], to investigate their utility and reliability in genotyping alleles that are within read length compared to more recent algorithms that consider a wider variety of read type evidence. The WGS data were aligned using two different aligners, Isaac [21] — an ultra-fast aligner, and Burrows-Wheeler Aligner (BWA)-MEM [22] — which is widely used in WGS studies [23], to see if the choice of aligner would influence the performance of STR methods.

First, we tested the classifier on results generated by the implementation of tools using default parameter settings. We then tweaked several parameters to optimize the sensitivity and specificity of STR tools included in this study. Once we established the parameters that yielded the best results, we used data generated with these settings to train and test the classifier and found a significant improvement in our model's ability to detect full-mutations compared to our default assessment. We then applied our decision tree model of STR algorithms to screen for expansions in known disease STR loci in ES and WGS data of 301 families (patient-parent trios (patient and both biological parents) or quads (patient, similarly affected sibling, and both biological parents)) with a proband who is suspected clinically to have a genetic disorder.

\section{Methods}

\section{WGS datasets with a known repeat expansion}

The WGS datasets with a known repeat expansion analyzed in this study include the BWA and Isaac alignments of: (1) the European Genome-phenome Archive (EGA) dataset [10] (EGAD00001003562), which consisted of data from 118 PCR-free WGS of Coriell samples, each with an $A R, A T N 1, A T X N 1, A T X N 3, D M P K$, $F M R 1, F X N$, or $H T T$ expansion (see Additional file 1: Table S1), and (2) C9orf72, FMR1, or FMR2 expansions of varying repeat lengths simulated using the ART NGS read simulator [24] (Additional file 1: Table S2). The simulated WGS data were included in our analysis to assess the performance of STR callers on expansions of extremely high GC content (100\%) that may be refractory to detection.

\section{Patient cohorts and ES and WGS data generation}

The patient cohorts screened for known STR expansions in this study consist of the ES data of 141 trios or quads from the Clinical Assessment of the Utility of Sequencing and Evaluation as a Service (CAUSES) Study [25] and the WGS data of 160 trios or quads from the Integrated Metabolomics And Genomics In Neurodevelopment (IMAGINE) [26] or CAUSES Studies. Subjects enrolled in the CAUSES Study were children who were suspected on clinical grounds to have a single gene disorder but in whom conventional testing had not identified a genetic cause. Subjects enrolled in the IMAGINE Study had impairment of motor function with onset before birth or within the first year of life and additional clinical features that made perinatal complications such as hypoxia or intracranial hemorrhage an unlikely explanation for their symptoms. The CAUSES and IMAG INE Study subjects were enrolled at the Children's and Women's Health Centre (Vancouver, British Columbia, Canada), and most of the enrolled subjects in both studies had intellectual disability. The ES or WGS data from the unaffected parents were used to verify the inheritance or unstable transmission of variants. The CAUSES and IMAGINE Studies were approved by the Institutional Review Board of the Children's and Women's Health Centre of British Columbia and the University of British Columbia (H15-00092 and H16-02126, respectively).

The trio/quad ES data were sequenced by Ambry Genetics (Aliso Viejo, USA), Centogene (Rostock, Germany), or Canada's Michael Smith Genome Sciences Centre (Vancouver, Canada) to a mean coverage of $\sim 60 \times$. The library preparation protocols and sequencers used to generate the trio/quad ES data are described in Additional file 2: Table S3.

The median coverage of the trio/quad WGS data ranged from 36 to $80 \times$ and was generated by the McGill University and Genome Quebec Innovation Centre (Quebec, Canada) or Canada's Michael Smith Genome Sciences Centre. WGS libraries were prepared using the NxSeq ${ }^{\oplus}$ AmpFREE Low DNA Library Kit Library Preparation Kit and Adaptors (Lucigen, Wisconsin, US) or xGen Dual Index UMI Adapters (Integrated DNA Technologies, Coralville, US) and sequenced on an Illumina HiSeqX sequencer.

The paired-end reads (125 or $150 \mathrm{bp}$ ) of both ES and WGS datasets were aligned to the UCSC hg19 human reference genome using BWA-MEM, and duplicates were marked with Picard [27]. All patient ES data underwent single-nucleotide variant (SNV) and indel analysis, and 140 out of the 141 trios or quads included in this 
study had no clinically relevant SNV/indel variants. We also analyzed the ES data of a quad with known myotonic dystrophy (type 1; DM1-MIM 160900) in the proband and his mother as a positive control. Our patient WGS data underwent SNV, indel, structural, and mitochondrial variant analysis. We have diagnosed a genetic disease in 44 of 85 (52\%) trios/quads studied in the CAUSES Study. Similarly, we have diagnosed a genetic disease in 46 of 88 (52\%) trios/quads studied in the IMAGINE Study to date. The WGS data of all trios/ quads from both CAUSES and IMAGINE Studies were included in this study.

\section{Bioinformatics tools for STR analysis}

The STR analysis tools implemented in this study include lobSTR [15], HipSTR [16], RepeatSeq [17], TREDPARSE [1], EH [10, 13], GangSTR [14], STRetch [11], and exSTRa [12]. The key features of these tools and the commands and parameters used to execute them are described in Table 1 and Additional file 2: Table S4, respectively. The two versions of EH v2 [10] and v3 [13] implement different algorithms. While we found EH_v2 to be easily tailorable for other STR loci, EH_v3 facilitates the analysis of STRs with complex or mixed repeat motifs. Therefore, we evaluated both versions of $\mathrm{EH}$.

\section{Disease STR catalogs}

The STR analysis tools assess known disease STRs included within a pre-defined STR catalog that contains repeat coordinate/motif information supplied by the tools' developers. The known pathogenic STR loci included in these catalogs, as well as their allelic categories and corresponding repeat lengths, are summarized in Additional file 2: Table S5. Notably, the region files for EH only included pre-defined OTS for FMR1 and C9orf72 loci, while GangSTR included OTS in the region files of all 12 pathogenic STR loci provided with the tool. Some of the known disease STRs analyzed in this study (AR, ATN1, FXN, and FMR2) were missing for GangSTR. Therefore, we added these loci and included their OTS as described in Mousavi et al. [14].

\section{Interpretation of full-mutations and non-full-mutations}

The data from the genotyping methods were classified as "full-mutation" if the estimated repeat lengths of the STRs exceeded their respective full-mutation thresholds (Additional file 2: Table S5). STRetch and exSTRa calls were classified as "full-mutation" if the $p$-values postmultiple-testing-adjustment were less than $<0.05$. For STRetch, we used the control file (containing data from 143 healthy individuals) provided with the tool.

\section{Negative control cohort}

Our negative control cohort included 100 individuals (healthy parents of affected children) from the IMAG INE Study who did not harbor expanded disease STR alleles based on the genotype calls from TREDPARSE, EH, and GangSTR.

\section{Decision tree classification}

Decision tree analysis is a supervised machine learning classification method [28]. We employed this approach to infer the best model or best combination of STR analysis tools to detect full-mutation expansions with optimal sensitivity and specificity. We used the Python scikit-learn machine learning library [29] to implement the decision tree classifier and used STR calls from EGA WGS to train and test the classifiers on data from Isaac and BWA alignments.

For our preliminary decision tree analysis, we used the outputs generated using the default parameters for each of the STR analysis tools. We compiled the results generated by the STR analysis tools on Isaac- and BWA-aligned WGS data. We labeled each EGA genome's true STR expansion status or class label as full-mutation or non-fullmutation for a given locus. The single known or characterized STR expansion in each of the EGA and simulated genomes was assigned to the "full-mutation" class, while the status of the other STR loci was assigned to "non-fullmutation". The data from STR callers were then transformed into binary flags: 1 indicating at least one of the two alleles was called as "full-mutation", and 0 indicating both alleles were "non-full-mutation". From there, we removed all rows with missing values and supplied the data to the classifier. We divided our dataset into 80 and $20 \%$ to train and test the classifier, respectively.

We used the Gini index approach to ascertain the efficiency of an attribute (i.e., the STR caller) in differentiating samples belonging to the full-mutation and non-full-mutation classes. To evaluate the performance of the classifier, we extracted different metrics, including precision (true positives $[\mathrm{TP}] /(\mathrm{TP}+$ false positives $[\mathrm{FP}]))$, recall $(\mathrm{TP} /(\mathrm{TP}+$ false negatives $[\mathrm{FN}]))$, accuracy $((\mathrm{TP}+$ true negatives $[\mathrm{TN}]) /(\mathrm{TP}+$ $\mathrm{TN}+\mathrm{FP}+\mathrm{FN}))$, and F1-score $(2 \times(($ precision $\times$ recall $) /($ precision + recall $))$ ), and analyzed the receiver operating characteristic (ROC) curve, a ratio of sensitivity $(\mathrm{TP} /(\mathrm{TP}+\mathrm{FN}))$ and inverted specificity $(1-$ $(\mathrm{TN} /(\mathrm{TN}+\mathrm{FP})))$, and precision-recall curve, a ratio of precision and recall or sensitivity.

To identify the best analysis model, we first selected important features using the Exhaustive Feature Selector algorithm from the machine learning extensions (MLxtend) Python library [30], which assessed the performance of all possible combinations of STR tools on 50 train-validation splits generated by five repeats of stratified 10-fold cross- 
validation on the training dataset. We then chose the best feature subset with the highest mean ROC_AUC (area under the curve) and tuned the hyper-parameters of the decision tree using the scikit's GridSearchCV on the training dataset for improved predictions.

We next ascertained whether tweaking some of the parameters would improve the performance of the STR analysis tools and the resultant decision tree model. First, we assessed the performance of EH with OTS on selected STR loci that are known to harbor expansions exceeding sequencing fragment lengths to retrieve unmapped and mismapped IRR/IRR pairs and improve the repeat length estimation and detection of full-mutations. Second, we used the intermediate repeat length threshold instead of the full-mutation threshold for the FMR1 locus to classify expanded alleles and documented the sensitivity as well as the false-positive rates of the genotypers. Third, we tested exSTRa's performance with control data from our negative control cohort. We carefully evaluated how these parameter tweaks influenced the performance of the STR analysis tools and selected the optimized outcomes to reimplement our decision tree classification analysis as mentioned above, and compared the results to our preliminary decision tree analysis with default parameters.

\section{Screening for known disease STR expansions in patient data}

Finally, we screened our patient trio/quad ES and WGS data for known disease STR expansions using the tools identified by the classifier. Of the probands analyzed in this study, 60 have had a clinical FMR1 STR testing, three have had clinical spinocerebellar ataxia (SCA) STR panel tests, one has had a clinical FXN STR test, and four have had a clinical DMPK STR test. All of these clinical PCR-based STR tests were negative for a pathogenic expansion, except for a confirmed DMPK fullmutation in a proband and his mother. All individuals who were expansion-negative at the tested locus were used as negative controls.

For all expanded STRs identified in patients, we analyzed the parental genotype calls to verify the inheritance or unstable transmission of the alleles. Subjects with potential expansions of known disease STRs were identified for orthogonal validation to ascertain the specificity of our decision tree. Molecular testing (PCR and capillary electrophoresis) of some of the identified STR candidates was performed by Centogene (Germany).

\section{Results}

Performance of STR algorithms on Isaac- versus BWAaligned WGS data

The lobSTR [15], HipSTR [16], RepeatSeq [17], EH versions 2 [10] and 3 [13], GangSTR [14], TREDPARSE [1],
STRetch [11], and exSTRa [12] results of Isaac- and BWA-aligned EGA and simulated FMR2 and C9orf72 WGS data are shown in Additional file 2: Table S6 and S7, respectively.

Of the known normal alleles $(\mathrm{n}=94)$ in EGA/simulated Isaac WGS, GangSTR, TREDPARSE, EH_v2, and EH_v3 correctly genotyped > 89\% of normal alleles (Additional file 2: Table S8). lobSTR, HipSTR, and RepeatSeq, on the other hand, correctly genotyped $30-75 \%$ of normal alleles; however, these tools were generally inconsistent in genotyping normal alleles of the C9orf72, FMR1, FMR2, FXN, and/or HTT loci. Of the known FMR1 intermediate and premutation alleles (n = 21), EH_v2 and EH_v3 correctly identified 18 and 16 alleles, respectively, followed by TREDPARSE (eight alleles) and GangSTR (six alleles) (Additional file 2: Table S9). lobSTR, HipSTR, and RepeatSeq either under-sized all known intermediate/premutation alleles or did not genotype them. We observed a similar trend among genotyped normal and intermediate/premutation alleles in BWA data, except that GangSTR did not correctly identify any intermediate/premutation alleles. As they only rely on spanning reads, lobSTR, HipSTR, and RepeatSeq did not detect any full-mutations in either Isaac- or BWA-aligned WGS data.

The sensitivity of EH_v2, EH_v3, GangSTR, TREDPA RSE, STRetch, and exSTRa run with default parameters to detect full-mutations across the different analyzed STR loci in Isaac- and BWA-aligned WGS is summarized in Table 2. EH_v2, EH_v3, TREDPARSE, and STRetch exhibited consistent performance, with a sensitivity of $\sim 70 \%$ in both Isaac and BWA alignments. GangSTR's sensitivity was better on Isaac (55\%) compared to BWA (38\%) alignments. In marked contrast, exSTRa detected more fullmutations for BWA (88\%) alignments compared to Isaac (56\%) (Additional file 3: Fig S1 for exSTRa's plots on Isaac- and BWA-aligned WGS). On Isaac-aligned data, STRetch, EH_v2, and EH_v3 detected the most fullmutations, followed by TREDPARSE, exSTRa, and GangSTR. On BWA-aligned data, exSTRa detected the most full-mutations, followed by STRetch, EH_v2, EH_v3, TREDPARSE, and GangSTR. Notably, although exSTRa and STRetch detected more full-mutations, they also had the most false-positive calls.

All full-mutations missed by genotypers were undersized and classified incorrectly as premutation, intermediate, or normal (Additional file 2: Table S10). Among the analyzed STR loci, FMR1, FMR2, and homozygous FXN full-mutations were particularly refractory to detection.

\section{Simulated versus real genomes}

We next implemented the STR callers on FMR1 simulations (13 BWA-aligned genomes simulated to harbor 
Table 2 Full-mutation samples detected in the EGA and simulated genomes using the default implementation of STR analysis tools

\begin{tabular}{|c|c|c|c|c|c|c|c|c|c|c|c|c|c|}
\hline Gene & $\begin{array}{l}A R \\
(\mathrm{n}=1)\end{array}$ & $\begin{array}{l}\text { ATN1 } \\
(\mathrm{n}=2)\end{array}$ & $\begin{array}{l}A T X N 1 \\
(\mathrm{n}=3)\end{array}$ & $\begin{array}{l}\text { ATXN3 } \\
(\mathrm{n}=1)\end{array}$ & $\begin{array}{l}\text { C9orf72 } \\
(\mathrm{n}=3)\end{array}$ & $\begin{array}{l}\text { DMPK } \\
(n=17)\end{array}$ & $\begin{array}{l}\text { FMR1 } \\
(\mathrm{n}=18)\end{array}$ & $\begin{array}{l}\text { FMR2 } \\
(\mathrm{n}=3)\end{array}$ & $\begin{array}{l}F X N \\
(\mathrm{n}=25)\end{array}$ & $\begin{array}{l}H T T \\
(n=13)\end{array}$ & FPs & $\begin{array}{l}\text { Total FM } \\
\text { detected }\end{array}$ & Sensitivity \\
\hline $\begin{array}{l}\text { FM } \\
\text { threshold } \\
\text { (rpts) }\end{array}$ & 37 & 47 & 38 & 59 & 60 & 50 & 200 & 200 & 65 & 39 & & & \\
\hline $\begin{array}{l}\text { Allelic } \\
\text { classification }\end{array}$ & $\mathrm{FM}$ & $\mathrm{FM}$ & FM & FM & FM & $\mathrm{FM}$ & FM & FM & $\begin{array}{l}\text { NL/FM or } \\
\text { FM/FM }\end{array}$ & $\begin{array}{l}\text { NL/FM or } \\
\text { FM/FM }\end{array}$ & & & \\
\hline \multicolumn{14}{|l|}{ Isaac } \\
\hline EH_V2 & 1 & 2 & 2 & 1 & 3 & 17 & 1 & 0 & 25 & 13 & 6 & 65 & 0.75581395 \\
\hline EH_v3 & 1 & 2 & 3 & 0 & 3 & 17 & 0 & 0 & 25 & 13 & 5 & 64 & 0.74418605 \\
\hline GangSTR & 0 & 2 & 2 & 0 & 0 & 16 & 0 & 0 & 16 & 11 & 8 & 47 & 0.54651163 \\
\hline TRED & 1 & 2 & 1 & 0 & 3 & 17 & 0 & 0 & 25 & 13 & 3 & 62 & 0.72093023 \\
\hline STRetch & 1 & 2 & 3 & 1 & 3 & 17 & 2 & 3 & 20 & 13 & 26 & 65 & 0.75581395 \\
\hline exSTRa & 1 & 2 & 3 & 0 & 3 & 17 & 1 & 3 & 5 & 13 & 33 & 48 & 0.55813953 \\
\hline \multicolumn{14}{|l|}{ BWA } \\
\hline EH_v2 & 1 & 2 & 2 & 1 & 3 & 17 & 0 & 0 & 25 & 13 & 6 & 64 & 0.74418605 \\
\hline EH_v3 & 1 & 2 & 3 & 0 & 3 & 17 & 0 & 0 & 25 & 13 & 5 & 64 & 0.74418605 \\
\hline GangSTR & 1 & 2 & 2 & 1 & 1 & 16 & 0 & 0 & 0 & 10 & 8 & 33 & 0.38372093 \\
\hline TRED & 1 & 2 & 1 & 0 & 3 & 17 & 0 & 0 & 25 & 13 & 10 & 62 & 0.72093023 \\
\hline STRetch & 1 & 2 & 3 & 1 & 3 & 17 & 2 & 3 & 20 & 13 & 26 & 65 & 0.75581395 \\
\hline exSTRa & 1 & 2 & 3 & 1 & 3 & 16 & 9 & 3 & 25 & 13 & 35 & 76 & 0.88372093 \\
\hline
\end{tabular}

The analyzed dataset had 86 samples with at least one known full-mutation allele. The number of true-positives detected by the tools, sensitivity, and the number of false positives identified in our default analysis of the Isaac- (top panel) and BWA-aligned (bottom panel) genomes are shown. NL normal, FM full-mutation, FPs false-positives, rpts repeats, EH_v2 ExpansionHunter version 2, EH_v3 ExpansionHunter version 3, TRED TREDPARSE

normal-full-mutation FMR1 alleles) with repeat counts similar to those of the fragile $\mathrm{X}$ reference samples in the EGA dataset to investigate whether GC-bias in experimental WGS is reflected in simulated data as well (Additional file 2: Table S11). All STR callers performed significantly better on simulated WGS with premutation and full-mutation FMR1 expansions compared to real data. Upon investigating the read-support evidence from $\mathrm{EH}$, we found that simulated WGS with premutation and full-mutation expansions had more IRRs in comparison to real WGS, demonstrating that simulated data of GC-rich repeat expansions could artificially inflate the accuracy and performance of STR callers. Subsequently, we excluded simulated genomes from the analysis.

\section{Decision tree classification}

We first trained and tested the decision tree classifier on the generated default parameter results of EH_v2, EH v3, GangSTR, TREDPARSE, STRetch, and exSTRa in the EGA genomes. After removing the rows with missing values, the compiled STR calls of Isaac- and BWAaligned WGS datasets had 1178 and 1176 rows (one row per sample per STR locus), respectively. We identified EH_v2, GangSTR, and exSTRa as the best feature set in Isaac data, tuned the hyper-parameters of the classifier with selected features, and implemented the model with the best hyper-parameters and features on test data. Additional file 3: Fig S2 shows the decision tree with EH_v2, which performed the best (had the lowest Gini impurity) in classifying alleles, assigned to the root node (node \#0). In the test data, the decision tree model had precision, recall, and F1-score of 100, 83, and 91\%, respectively, to detect full-mutations; for non-fullmutations, the precision, recall, and F1-score were 99, 100, and 99\%, respectively (Additional file 3: Fig S3a). The ROC and precision-recall plots are shown in Additional file 3: Fig S3b and the confusion matrix showing the classification of full-mutations and non-fullmutations in the test dataset is shown in Additional file 3: Fig S3c.

In BWA-aligned data, the best model included EH_v3, STRetch, exSTRa, GangSTR, and TREDPARSE. EH_v3 at the root node correctly classified 45 out of 62 fullmutations and 873 out of 878 non-full-mutations in the training dataset, with exSTRa and GangSTR recovering one of the full-mutations missed by EH_v3 (Additional file 3: Fig S4). The precision, recall, and F1-score to detect full-mutations and non-full-mutations in the test data were 100,89 , and $94 \%$ and 99,100 , and $100 \%$, respectively (Additional file 3: Fig S5a). The ROC and precision-recall curves and confusion matrix are shown in Additional file 3: Fig S5b and S5c. 
In Isaac and BWA analyses, three and five of the six features (i.e., STR tools) contributed to the performance of the model, led by EH_v2 and EH_v3, respectively (Additional file 3: Fig S3d and S5d). The sensitivity for detecting full-mutations in BWAaligned data was slightly higher compared to the Isaac analysis. Overall, the decision tree classifier on Isaac and BWA test data using the default analysis missed $\sim 10$ to $20 \%$ of full-mutations. To improve the detection sensitivity, we evaluated some parameters that we believed might help capture more of the true fullmutations.

\section{Tested parameters}

First, we tested the effect of including OTS in the detection of full-mutations. While GangSTR's region files included OTS for all analyzed loci, the authorsupplied JSON files of EH did not include OTS for $D M P K$ and FXN loci, which are known to harbor expansions exceeding fragment lengths. In our initial $\mathrm{EH}$ run without OTS, we noted reduced sensitivity in the detection of FXN full-mutations (Additional file 2: Table S12). Therefore, we added OTS for analyzing these loci with EH_v2. There was no improvement in sensitivity, highlighting the general limitation of the genotypers in reliably detecting homozygous FXN full-mutation expansions.

Second, because GC-rich expansions such as those at the FMR1 locus tend to be under-sized owing to reduced coverage even in PCR-free Illumina WGS datasets [10], we used an intermediate repeat length threshold (54 repeats) for FMR1 instead of their full-mutation threshold (200 repeats). With this tweak, EH_v2 and EH_v3 detected all FMR1 full-mutations in Isaac- as well as BWA-aligned data (Table 3). TREDPARSE detected 83-89\% of FMR1 full-mutations, while GangSTR detected $16-22 \%$ of them. The identified false positives in this analysis include the known FMR1 premutations and a few borderline FMR1 intermediate alleles that are closer to the threshold.

Lastly, we hypothesized that adding data from a control cohort to exSTRa's analysis would further improve its full-mutation detection sensitivity. In Isaac alignments, exSTRa's sensitivity improved moderately for detecting FXN full-mutations, but no improvements were observed in full-mutation detection at other loci (Additional file 3: Fig S6a). In BWA alignments, exSTRa yielded better sensitivity with controls and detected all homozygous FXN full-mutation expansions, as well as all FMR1 full-mutations (Additional file 3: Fig S6b).

Of these parameters, using the intermediate threshold for FMR1 genotype analysis and performing exSTRa's analysis with controls were useful in detecting refractory STR expansions. We provided these improved results to the classifier. In Isaac data, feature selection and hyperparameter tuning helped identify TREDPARSE, STRetch, and EH_v3 as the best feature subset (Additional file 3: Fig S7). In BWA data, the best feature subset included STRetch, EH_v3, and exSTRa (Fig. 1a). The classifier's precision, recall, and F1-score in Isaac- and BWA-aligned test datasets were 90,100 , and $95 \%$ and 82,100 , and $90 \%$ to detect full-mutations and 100, 99, and $100 \%$ and 100 , 98, and $99 \%$ to detect non-full-mutations, respectively (Additional file 3: Fig S8a, and Fig. 1b). The ROC and precision-recall curves are shown in Additional file 3: Fig S8b and Fig. 1c, and the confusion matrix in Additional file 3: Fig S8c and Fig. 1d. The feature importances of the three selected STR analysis tools for Isaac- and BWA-aligned WGS are shown in Additional file 3: Figure S8d and Fig. 1e. Among the STR tools, TREDPARSE and STRetch ranked first in Isaac and BWA alignments, respectively. This model with the optimized parameters performed better than the default analysis, detecting all full-mutations in both Isaac and BWA test data.

Table 3 FMR1 and FMR2 full-mutations detected by ExpansionHunter, GangSTR, and TREDPARSE with lowered repeat length threshold

\begin{tabular}{|c|c|c|c|c|c|c|c|c|c|c|c|c|c|c|}
\hline Aligner & Isaac & & & & & & & BW & & & & & & \\
\hline Locus & FMR & $1=1$ & & & FMF & $=3)$ & & $\overline{F M F}$ & $=1$ & & & $F M F$ & $=3)$ & \\
\hline FM threshold & $54 \mathrm{rc}$ & & & & $60 r$ & & & 54 & & & & $60 \mathrm{r}$ & & \\
\hline Allelic classification & $\overline{F M}$ & IM & $\mathrm{NL}$ & $\overline{\text { FP }}$ & $\overline{\mathrm{FM}}$ & NL & $\overline{F P}$ & $\overline{\mathrm{FM}}$ & IM & $\mathrm{NL}$ & $\overline{\mathrm{FP}}$ & $\overline{\mathrm{FM}}$ & $\mathrm{NL}$ & FP \\
\hline$\overline{E H \_v 2}$ & 18 & . & . & 20 & 3 & . & 0 & 18 & . & . & 16 & 3 & . & 0 \\
\hline EH_v3 & 18 & . & . & 22 & 3 & . & 0 & 18 & . & . & 22 & 3 & . & 0 \\
\hline GangSTR & 4 & . & 14 & 7 & 0 & 3 & 0 & 3 & & 15 & 0 & 0 & 3 & 0 \\
\hline TREDPARSE & 15 & 1 & 2 & 8 & 0 & 3 & 0 & 16 & & 2 & 13 & 0 & 3 & 0 \\
\hline
\end{tabular}

The number of full-mutations (FMs) misclassified as normal (NL; < 45 repeats for FMR1 and < 31 repeats for FMR2) or intermediate (IM; $45-54$ repeats for FMR1) allele are shown. The true number ( $\mathrm{n}$ ) of known FM alleles in the FMR1 and FMR2 genes is indicated in parenthesis. False-positive (FP) calls made by the tools are also reported 


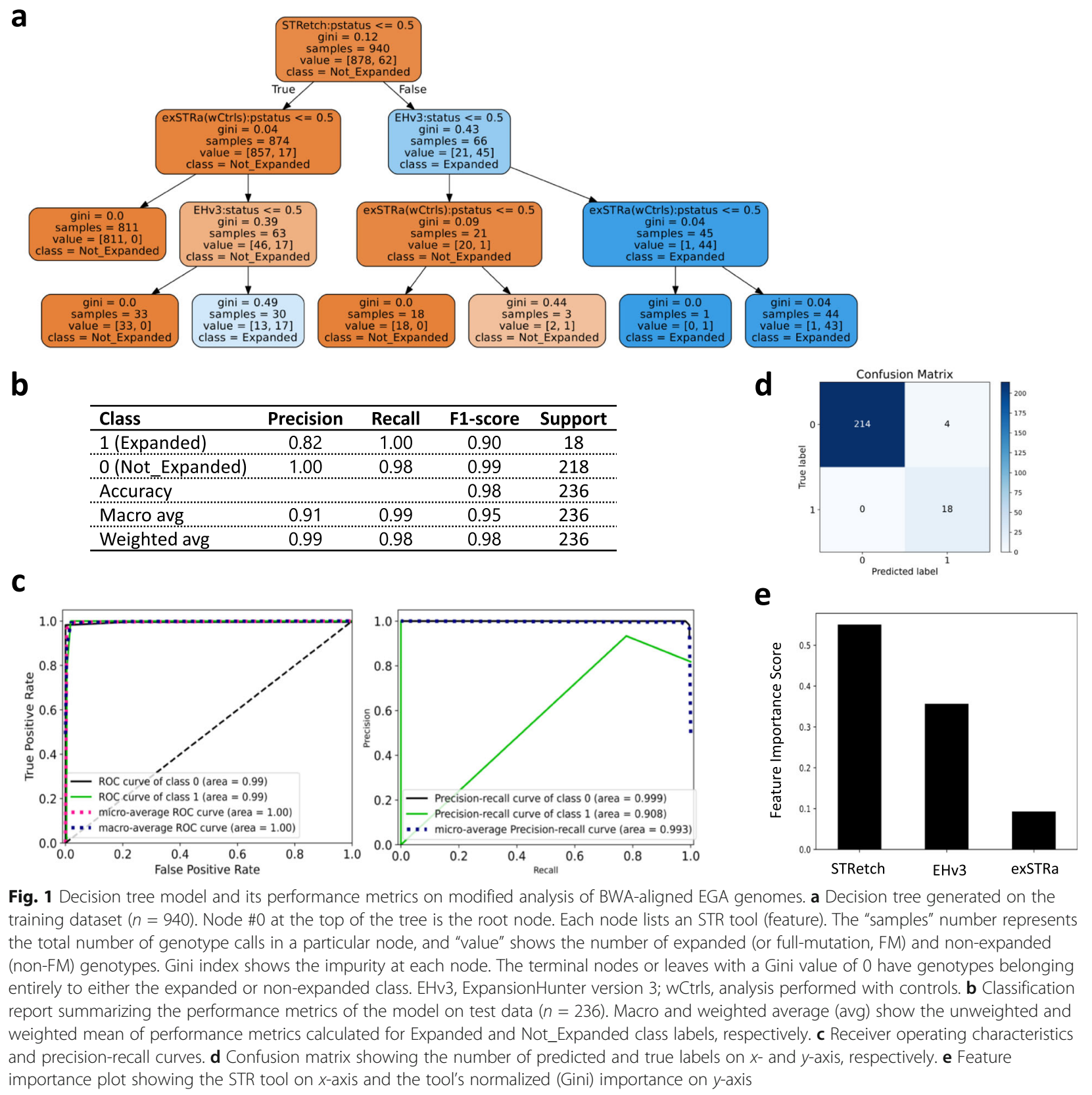

\section{Analysis of known disease STRs in clinical NGS data}

The decision rules that emerged from the modified analyses suggest the best approach to categorizing fullmutations in BWA data is to use STRetch, EH_v3, and exSTRa (see Fig. 1). Applying this pipeline on our BWAaligned patient ES and WGS data, we identified 21 samples of interest, 10 with full-mutation expansions of the $A R, A T X N 1, A T X N 8, D M P K, F X N$, or HTT locus, two with borderline ATXN2 alleles, and seven with intermediate or premutation alleles in the FMR1 locus (summarized in Table 4). Additional file 2: Table S13 shows the EH_v3, STRetch, and exSTRa results of the identified STR candidates.

We found that all probands with an identified STR candidate inherited the allele from a parent (Additional file 2: Table S13). The inherited expansions either remained unchanged, decreased by one or a few repeat units, or increased by 1 to $\sim 15$ repeats during intergenerational transmission.

All individuals who tested negative in their clinical molecular assessments for FMR1, FXN, SCA, or DMPK full-mutation expansions were also categorized as non- 
Table 4 Short tandem repeat candidates identified in our patient cohort

\begin{tabular}{|c|c|c|c|c|c|c|c|}
\hline $\begin{array}{l}\text { Sample } \\
\text { ID }\end{array}$ & Gene & Inheritance & Sequencing & $\begin{array}{l}\text { Pathogenic } \\
\text { SNV/indel/SV } \\
\text { finding }\end{array}$ & Phenotype & $\begin{array}{l}\text { STR } \\
\text { finding }\end{array}$ & $\begin{array}{l}\text { Molecular } \\
\text { validation }\end{array}$ \\
\hline $1901-P$ & $A R$ & Inherited & WGS & No & $\begin{array}{l}\text { Short stature, delayed gross motor, speech and language development, } \\
\text { spasticity, cerebral palsy, and hypertonia }\end{array}$ & $\begin{array}{l}\text { FM (full- } \\
\text { penetrance) }\end{array}$ & $\begin{array}{l}\text { FM (reduced/ } \\
\text { full-penetrance) }\end{array}$ \\
\hline $1901-\mathrm{F}$ & $A R$ & . & WGS & & & $\begin{array}{l}\text { FM (full- } \\
\text { penetrance) }\end{array}$ & $\begin{array}{l}\text { FM (reduced/ } \\
\text { full-penetrance) }\end{array}$ \\
\hline $532-M$ & ATXN1 & & WGS & & & $\begin{array}{l}\text { FM (full- } \\
\text { penetrance) }\end{array}$ & n.a. \\
\hline $821-P$ & ATXN2 & Inherited & ES & No & $\begin{array}{l}\text { Mild intellectual disabilities, systemic hypertension, cutis aplasia, } \\
\text { congenital heart defect, and limb anomalies }\end{array}$ & Borderline $e^{a}$ & n.a. \\
\hline 821-M & ATXN2 & & ES & . & & Borderline $^{a}$ & n.a. \\
\hline 1099-P & ATXN8 & Inherited & ES & No & $\begin{array}{l}\text { Hearing loss, cataract, myopia, visceral (kidney and spleen) cysts, } \\
\text { proteinuria, and dysmorphic facial features }\end{array}$ & $\begin{array}{l}\text { FM (higher } \\
\text { penetrance) }\end{array}$ & n.a. \\
\hline $1099-M$ & ATXN8 & & ES & & & $\begin{array}{l}\text { FM (higher } \\
\text { penetrance) }\end{array}$ & n.a. \\
\hline $235-\mathrm{P}$ & ATXN8 & Inherited & WGS & No & Mild to moderate intellectual disability, and psychosis & $\begin{array}{l}\text { FM (higher } \\
\text { penetrance) }\end{array}$ & n.a. \\
\hline $235-M$ & ATXN8 & & WGS & & & $\begin{array}{l}\text { FM (higher } \\
\text { penetrance) }\end{array}$ & n.a. \\
\hline 2010-P & DMPK & Inherited & ES & Definite & $\begin{array}{l}\text { Myotonic dystrophy type 1, inguinal hernias, joint hypermobility, } \\
\text { strabismus, mild intellectual disability, and dysmorphic facial features }\end{array}$ & $\begin{array}{l}\text { FM (full- } \\
\text { penetrance) }\end{array}$ & $\begin{array}{l}\text { FM (full- } \\
\text { penetrance) }\end{array}$ \\
\hline $2010-M$ & DMPK & & ES & & Myotonic dystrophy type 1 & $\begin{array}{l}\text { FM (full- } \\
\text { penetrance) }\end{array}$ & $\begin{array}{l}\text { FM (full- } \\
\text { penetrance) }\end{array}$ \\
\hline $148-M$ & FMR1 & . & WGS & & & PM & $\begin{array}{l}\text { n.a. (proband is } \\
\text { negative for } \\
\text { FMR1 FM) }\end{array}$ \\
\hline $800-\mathrm{F}$ & FMR1 & & WGS & & & IM & n.a. \\
\hline 480- $P$ & FMR1 & Inherited & WGS & Probable & $\begin{array}{l}\text { Moderate intellectual disability, language delay, autism, borderline } \\
\text { macrocephaly, low set ears, down slanting palpebral fissures, high } \\
\text { palate, and soft skin }\end{array}$ & PM & n.a. \\
\hline $712-M$ & FMR1 & & WGS & & & PM & $\begin{array}{l}\text { n.a. (proband is } \\
\text { negative for } \\
\text { FMR1 FM) }\end{array}$ \\
\hline $925-\mathrm{P}$ & FMR1 & Inherited & WGS & No & $\begin{array}{l}\text { Intellectual disability, developmental delay including speech delay, } \\
\text { dysmorphic features, and behavioral challenges }\end{array}$ & PM & Negative for FM \\
\hline $925-\mathrm{S}$ & FMR1 & Inherited & WGS & No & $\begin{array}{l}\text { Intellectual disability, autism, developmental delay, and dysmorphic } \\
\text { features }\end{array}$ & IM & n.a. \\
\hline $925-M$ & FMR1 & & WGS & & & PM & n.a. \\
\hline 1987-F & FXN & & WGS & . & & NL/FM & $\begin{array}{l}\text { Heterozygous } \\
\text { NL/FM carrier }\end{array}$ \\
\hline 1530-P & $H \pi T$ & Inherited & WGS & Uncertain & $\begin{array}{l}\text { Global developmental delay, seizures, gliosis, developmental regression, } \\
\text { encephalomalacia, hirsutism, nystagmus, optic atrophy, cyanosis, } \\
\text { abnormal muscle tone, scoliosis, hearing impairment, and otitis media }\end{array}$ & $\begin{array}{l}\text { FM } \\
\text { (reduced } \\
\text { penetrance) }\end{array}$ & $\begin{array}{l}\text { FM (reduced } \\
\text { penetrance) }\end{array}$ \\
\hline $1530-\mathrm{F}$ & $H T T$ & & WGS & & & $\begin{array}{l}\text { FM } \\
\text { (reduced } \\
\text { penetrance) }\end{array}$ & $\begin{array}{l}\text { FM (reduced } \\
\text { penetrance) }\end{array}$ \\
\hline
\end{tabular}

Probands with an identified STR candidate are given a "-P" suffix in the "Sample ID" column; sibling of the proband, "-S"; mother, "-M"; and father, "-F". The genes harboring the STR candidate identified by our bioinformatics workflow and the inheritance pattern deciphered by comparing the proband's STR call with that of the parents are reported. The "Sequencing" column shows the technology used: whole-genome sequencing (WGS) or exome sequencing (ES). The "Pathogenic SNV/indel/SV Finding" column indicates whether the proband has had a definite, probable, certain, or no diagnosis of a single-nucleotide variant (SNV), indel, or structural variant (SV). Phenotypic presentations reported in the probands, STR finding from our bioinformatics analysis, and the results from the molecular validation (if available) are also presented. NL normal, IM intermediate, PM premutation, FM full-mutation, n.a. not available

${ }^{a}$ Reduced-penetrance alleles have 33-34 repeats and full-penetrance alleles have $\geq 37$ repeats

expanded by our bioinformatics workflow (data not shown). In the ES data of a proband (2010-P) and his mother (2010-M) who had DM1 with DMPK fullmutation (> 50 repeats) findings upon prior molecular assessment, EH_v3 and exSTRa identified the fullmutation expansion. However, the repeat length estimated by EH_v3 in 2010-P and 2010-M was 50 repeats, which is significantly lower than the molecular findings of 150 repeats in 2010-P and 430 repeats in 2010-M (Additional file 2: Table S13).

Based on the repeat lengths estimated by EH_v3, we categorized the identified full-mutations as 
reduced- or full-penetrance (Table 4; the different repeat size ranges associated with reduced- and fullpenetrance of the STR expansion disorders are summarized in Additional file 2: Table S5). Six of the full-mutations we identified in the probands and parents were in the fully penetrant or higher penetrance repeat size range, while two were in the reducedpenetrance range.

We performed PCR-based molecular tests to verify the expansion status of a subset of the identified fullmutations (molecular findings summarized in the last column of Table 4 and Additional file 2: Table S13). The HTT full-mutations identified by EH_v3 (37 repeats), STRetch, and exSTRa in a proband (1530-P) and his father (1530-F) were concordant with the molecular results (37 \pm 1 repeats). The $A R$ full-mutations in a father (1901-F) and proband (1905-P) identified by EH_v3 (38 repeats) and STRetch were consistent with the PCR results (37 \pm 1 repeats). In addition, the $F X N$ candidate in a father (1987-F) identified by EH_v3 (69 repeats) was verified as full-mutation by PCR (87 \pm 1 repeats). However, the clinical PCR test could not determine the exact repeat length of this allele due to the presence of sequence interruptions. In two samples (1099-P and 1099M), EH_v3 did not return genotype calls for the ATXN8 locus, which appears to be expanded as per STRetch and exSTRa. Modifying the ATXN8 locus in the EH_v3 cata$\log$ to ["VariantType": "Repeat", "LocusId": "ATXN8", "LocusStructure": "(CTG)"”, "ReferenceRegion": "13: 70713516-70713560"\} helped with successful genotyping and identification of full-mutation with > 200 CTG repeats in both samples.

A TBP full-mutation identified by EH_v3 (54 repeats), but not supported by either STRetch or exSTRa, could not be verified by PCR $(37 \pm 1$ repeats) (data not shown). Closer inspection of EH's TBP genotype using GraphAlignmentViewer [31] revealed the lack of reads supporting the genotype call made in 1992-M. Visualization of candidate STRs, particularly those that were detected only by EH but not supported by either STRetch or exSTRa, may help reduce the number of false positives and confirmatory molecular tests required.

Lastly, we investigated the genotype calls of disease STRs made by EH_v2, EH_v3, and GangSTR in our patient ES and WGS datasets to see if the normal allele frequency distribution at these loci agreed with the reported population frequencies of normal alleles (Additional file 3: Fig S9 and S10, and Additional file 2: Table S14). In general, the repeat length distribution pattern of the STR alleles for most loci was consistent across the ES (Additional file 3: Fig S9) and WGS (Additional file 3: Fig S10) data, except for the FMR1 and FMR2 loci, which were characterized inconsistently in ES data. EH_ v3 genotyped fewer ATXN8 alleles and had a different repeat length distribution profile for the ATXN7 and HTT loci in ES data. For the CSTB locus, more 1-repeat genotype calls were made by the tools in ES data, while we found none in WGS data.

More than half of the individuals in our clinical cohort are of European ancestry, so we compared the frequency of the three most common alleles ascertained in WGS data to the common normal allele in the Caucasian population reported in the literature (Additional file 2: Table S14). Except for a few loci, the repeat lengths of most common alleles determined by the tools were generally in good agreement with the reported repeat length of the common normal alleles in the Caucasian population.

\section{Discussion}

The contribution of STR expansions to disease is just beginning to be understood. About 40 neurological disorders have been found to be caused by STR expansion mutations [2], with some recent studies reporting the identification of additional pathogenic STR expansion loci through NGS or the more advanced thirdgeneration long-read sequencing technologies [32-36]. While repeat expansion disorders typically have a delayed age of onset, pre-symptomatic individuals have been reported to exhibit subtle cognitive, behavioral, or structural changes in the brain $[37,38]$. Presymptomatic detection can help elucidate the pathophysiology and progression of the disease [39] and promote the development and timely implementation of disease-modifying interventions [40].

The challenges in detecting and characterizing repeat lengths of STR expansions in short-read NGS data are well recognized [41]. However, recent algorithmic improvements facilitate the detection of STR expansions that exceed read and/or fragment lengths, providing the opportunity to analyze a larger number of known disease STR loci simultaneously through ES or WGS [1, 10-14]. The analysis of STRs in clinical NGS data using these improved bioinformatics methods may help detect pathogenic full-mutation expansions and identify individuals with at-risk premutation, intermediate, or mutable normal alleles that may expand into an fullmutation in subsequent generations.

Of the available STR analysis tools, EH, GangSTR, and TREDPARSE are particularly valuable because they leverage evidence beyond reads that span an STR, enabling the genotyping of larger repeat expansions than is possible with spanning-read-only methods (lobSTR, HipSTR, and RepeatSeq). In this study, we have shown that EH, GangSTR, and TREDPARSE are also more reliable in genotyping STRs within read length compared to 
lobSTR, HipSTR, and RepeatSeq. STRetch and exSTRa can detect STR expansions but they do not reliably genotype them (STRetch) or do not genotype them at all (exSTRa). GangSTR and STRetch may also be useful in scanning the entire genome or exome for novel diseasecausing STR expansions because they are not limited to the analysis of a prespecified catalog of known disease STRs, as other tools are.

We have shown through a systematic analysis that the choice of aligner could impact the performance of the STR analysis tools. The differences in the number of unmapped reads and reads with zero mapping quality may explain some of these performance inconsistencies we noted comparing the STR outputs from Isaac- and BWA-aligned data (Additional file 3: Fig S11). Selecting methods that function optimally with a given aligner appears to be a critical first step in designing a clinical bioinformatics workflow to screen STR expansions in NGS data.

In-line with earlier studies $[10,12]$, we found the characterization of FMR1 full-mutations to be challenging. Homozygous FXN full-mutations were also refractory to detection because the genotypers typically identified only one of two full-mutation alleles. With our modified analysis, some of the tools detected more fullmutations at these loci. Notably, exSTRa performed better with an external control cohort, detecting all FXN and FMR1 full-mutations in BWA data. Also, reducing the repeat length thresholds from full-mutation to intermediate size range enabled the detection of FMR1 fullmutations with EH_v2, EH_v3, and TREDPARSE. With this reduced cut-off, the tools may also detect some intermediate and premutation carriers who, although are not affected, may be at risk of having children with fragile $\mathrm{X}$ syndrome if their intermediate/premutation allele is highly unstable and/or susceptible to late-onset conditions [42]. Early detection and genetic counseling of these at-risk individuals may, therefore, help intermediate/premutation allele carriers make informed reproductive decisions [42].

Our decision tree analyses favored using an ensemble approach that combined three of the most important STR methods (TREDPARSE, EH_v3, and STRetch for Isaac-aligned datasets, and STRetch, EH_v3, and exSTRa for BWA-aligned datasets). Comparing the best ensemble model with the stand-alone performance of the important tools revealed the advantage of adopting the ensemble approach (Additional file 2: Table S15). In BWA data, although exSTRa and EH_v3 alone had the same $100 \%$ recall as the ensemble model, the precision of individual tools was 4-24\% lower compared to the ensemble model, showing that the latter has the potential to reduce or eliminate false positives while maintaining high sensitivity. This is consistent with our clinical cohort analysis wherein a full-mutation in TBP locus identified only by EH_v3 could not be verified by PCR; however, seven full-mutations identified by EH_v3 together with STRetch and/or exSTRa were confirmed by clinical PCR tests (Additional file 2: Table S13).

Our ensemble pipeline of EH_v3, STRetch, and exSTRa takes $\sim 2.5 \mathrm{~h}$ per genome. Most methods, including EH_v3 and exSTRa, can perform targeted disease STR analysis on a single CPU within a matter of few seconds to minutes (Additional file 2: Table S16). STRetch took $2 \mathrm{~h}: 24 \mathrm{~min}$ per genome on 16 CPUs as it profiles a larger catalog of STRs $(\sim 300 \mathrm{k})$ genome-wide and performs statistical analysis to detect outliers.

For DMPK assessment with EH (the default catalog file of which does not include OTS), we recommend including OTS as this results in a significant improvement in the repeat length estimation, particularly in WGS data. Although the threshold for defining pathogenic DMPK full-mutations that cause DM1 is only 50 repeats and $\mathrm{EH}$ and other tools detect $D M P K$ full-mutations with $100 \%$ sensitivity, the different clinical forms of DM1 (mild, classic, and congenital), associated with varying severity and age of onset of symptoms are caused by $D M P K$ full-mutations in the ranges of 50- 150, 100$\sim 1000$, and $>1000$ repeat units, respectively [43]. We show that with OTS, EH performs better at sizing $D M P K$ full-mutations that ranged from $\sim 130$ to over 2000 repeats in the EGA WGS data and yields estimates that correlate better with full-mutation repeat lengths in these individuals (Additional file 3: Fig S12).

Although the methods we tested perform well in detecting and sizing full-mutations, for some disease STR loci, the difference between a non-full-mutation and a full-mutation, or between a reduced-penetrance and full-penetrance full-mutation is only a few repeat units, making it difficult to discriminate between these borderline alleles and their clinical significance. This limitation is also inherent to PCR-based tests as DNA polymerase slippage during STR amplification may result in underor over-estimation of STR size by one or two repeat units [44].

Using our approach, we were able to confirm the presence of a clinically validated $D M P K$ full-mutation in the ES data of a proband and his mother with DM1, inherited HTT and AR full-mutations in two families, and also the presence of an FXN normal/full-mutation in a father using clinical PCR and capillary electrophoresis. Importantly, none of the 67 individuals who previously had a negative clinical FMR1, FXN, SCA, or DMPK test result were falsely identified as "expanded" by our computational workflow.

Our analysis demonstrates that combining genotyping and statistical STR analysis tools yields optimal results. Of the currently available genotypers that can detect 
pathogenic expansions, only GangSTR works on STRs genome-wide. To identify expansions at novel loci, there is a need to expand the utility of existing genotypers or develop complementary genotyping approaches to interrogate candidates beyond the limited catalog of known disease STRs. The STR methods we used to analyze our clinical ES and WGS data were selected based on decision tree classification analysis on WGS. The main limitation with ES is its inconsistency in coverage and GC-bias, but we found that as long as there are sufficient reads mapping to the locus, the tools that work on WGS also perform reasonably well on ES data.

\section{Conclusions}

Clinical NGS datasets are typically screened for pathogenic SNVs, small indels, and SVs to diagnose the underlying cause of genetic disorders. However, STRs are often not analyzed due to the lack of a reliable and well-optimized computational pipeline. We aimed to demonstrate the utility of STR analysis methods as a first-tier screen for pathogenic expansions in clinical NGS data and to promote the rapid integration of STR callers into routine clinical genomic analyses. Through a thorough evaluation of eight existing STR callers (lobSTR, HipSTR, RepeatSeq, TREDPARSE, EH, GangSTR, STRetch, and exSTRa), we have outlined important factors, such as the choice of sequence aligners and parameter tweaks of STR callers, which can improve the sensitivity to detect pathogenic expansions. Using decision tree classification, we illustrated the best aligner-specific pipeline to detect STR expansions with optimal sensitivity and specificity. To demonstrate the utility of this pipeline, we screened the ES and WGS data of 301 patient-parent trios/quads and identified samples with full-mutation, borderline, and intermediate/premutation alleles. Clinical PCR confirmation of a subset of identified full-mutation expansions showed absolute concordance between molecular and bioinformatics findings. Our results show that the incorporation of our recommended pipeline of tools to analyze clinical ES and WGS data can reliably identify pathogenic STR expansions, which could promote cascade testing in affected families and improve the diagnostics, treatment, and management of repeat expansion disorders.

\footnotetext{
Abbreviations

AR: Androgen receptor; ATN: Atrophin; ATXN: Ataxin; AUC: Area under the curve; bp: Base pair; BWA: Burrows-Wheeler Aligner; C9orf72: Chromosome 9 open reading frame 72; CAUSES: Clinical Assessment of the Utility of Sequencing and Evaluation as a Service; DM: Myotonic dystrophy; DMPK: Dystrophia Myotonica Protein Kinase; EGA: European Genomephenome Archive; EH: ExpansionHunter; ES: Exome sequencing; FM: Fullmutation; FMR: Fragile X mental retardation; FN: False negative; FP: False positive; FXN: Frataxin; HTT: Huntingtin; IM: Intermediate; IMAGINE: Integrated Metabolomics And Genomics In Neurodevelopment; IRR: In-repeat reads; NGS: Next-generation sequencing; NL: Normal; OTS: Off-target sites; PCR: Polymerase chain reaction; PM: Premutation; ROC: Receiver operating
}

characteristic; SCA: Spinocerebellar ataxia; SNV: Single-nucleotide variant; STR: Short tandem repeat; TBP: TATA-box binding protein; TN: True negative; TP: True positive; WGS: Whole-genome sequencing

\section{Supplementary Information}

The online version contains supplementary material available at https://doi. org/10.1186/s13073-021-00932-9.

Additional file 1: Table S1: Experimental repeat size data of the samples in the Coriell European Genome-phenome Archive dataset. Table S2: Short tandem repeat alleles simulated in C9orf72, FMR1, and FMR2 genes using ART simulator.

Additional file 2: Table S3: CAUSES exome sequence data summary. Table S4: Commands and parameters used for the implementation of IobSTR, RepeatSeq, HipSTR, TREDPARSE, ExpansionHunter, STRetch, exSTRa, and GangSTR. Table S5: Summary of repeat loci in catalogs used by short tandem repeat analysis methods. Table S6: Short tandem repeat calls of ExpansionHunter, GangSTR, TREDPARSE, IobSTR, HipSTR, RepeatSeq, STRetch, and exSTRa in the Isaac-aligned European Genomephenome Archive and simulated genomes. Table S7: Short tandem repeat calls of ExpansionHunter, GangSTR, TREDPARSE, IobSTR, HipSTR, RepeatSeq, STRetch, and exSTRa in the BWA-aligned European Genomephenome Archive and simulated genomes. Table S8: Known normal alleles characterised by ExpansionHunter, GangSTR, TREDPARSE, lobSTR, HipSTR, and RepeatSeq in the European Genome-phenome Archive and simulated genomes. Table S9: Known FMR1 intermediate and premutation alleles characterised by ExpansionHunter, GangSTR, TREDPARSE, IobSTR, HipSTR, and RepeatSeq in the European Genome-phenome Archive and simulated genomes. Table S10: Full-mutation alleles characterised by ExpansionHunter, GangSTR, and TREDPARSE in the European Genome-phenome Archive and simulated genomes. Table S11: Performance of short tandem repeat callers on simulated and real genomes with FMR1 CGG-repeat expansions in normal, premutation, and full-mutation size ranges. Table S12: Analysis and detection of DMPK, FMR1, and FXN full-mutation alleles using ExpansionHunter version 2 and GangSTR with and without off-target sites. Table S13: ExpansionHunter, STRetch, and exSTRa calls and molecular validation results of the short tandem repeat candidates identified in the CAUSES and IMAGINE genomes and exomes, Table S14: Repeat length distributions of the genotyped AR, ATN1, ATXN1, ATXN2, ATXN3, ATXN7, ATXN8/ATXN80S, ATXN10, C9orf72, CACNA1A, CBL, CNBP, CSTB, DMPK, FMR1, FMR2, FXN, HTT, JPH3, NOP56, PHOX2B, $P P P 2 R 2 B$, and TBP alleles in the CAUSES and IMAGINE genomes. Table S15: Summary of performance metrics of the best model and individual STR tools in BWA modified analysis. Table S16: Run-time and peak memory requirements of short tandem repeat callers and aligners on a 40x human genome.

Additional file 3: Fig S1: exSTRa plots of EGA and simulated genomes. Fig S2: Decision tree model of the default analysis of Isaac-aligned EGA genomes on the training dataset. Fig S3: Performance metrics of the decision tree model in the default analysis of Isaac-aligned EGA genomes on the test dataset. Fig S4: Decision tree model of the default analysis of BWA-aligned EGA genomes on the training dataset. Fig S5: Performance metrics of the decision tree model in the default analysis of BWA-aligned EGA genomes on the test dataset. Fig S6: exSTRa plots of EGA genomes analyzed with 100 controls. Fig S7: Decision tree model of the modified analysis of Isaac-aligned EGA genomes on the training dataset. Fig S8: Performance metrics of decision tree model in the modified analysis of Isaac-aligned EGA test dataset. Fig S9: Allele frequency distribution of analyzed disease short tandem repeat loci in the CAUSES exomes. Fig S10: Allele frequency distribution of analyzed disease short tandem repeat loci in the CAUSES and IMAGINE genomes. Fig S11: Coverage and alignment statistics of Isaac- and BWA-aligned EGA genomes. Fig S12: Analysis of the DMPK locus by ExpansionHunter version 2 with and without offtarget sites in the EGA dataset.

\section{Acknowledgements}

We would like to thank all the CAUSES and IMAGINE Study investigators. CAUSES Study investigators include Shelin Adam, Christele Du Souich, Alison Elliott, Anna Lehman, Jill Mwenifumbo, Tanya Nelson, Clara van Karnebeek, 
and Jan Friedman. The CAUSES Study is funded by Mining for Miracles, British Columbia Children's Hospital Foundation, and Genome British Columbia. IMAGINE Study investigators include Patricia Birch, Madeline Couse, Colleen Guimond, Anna Lehman, Jill Mwenifumbo, Clara van Karnebeek, and Jan Friedman. We thank Compute Canada for the Research Allocation Competitions allocation, which facilitated our analysis of the IMAG INE and EGA genomes, and Julia Handra for coordinating the STR molecular testing of the clinical samples.

\section{Authors' contributions}

ISRB and JMF conceptualized the study. ISRB designed the experiments. ISRB and JJP carried out the experiments, generated the data, and prepared the tables and figures. ISRB, JJP, RC, IB, and JMF analyzed and interpreted the findings. $\mathrm{CL}$ and $\mathrm{IB}$ provided critical inputs on the design and implementation of the decision tree classifier. CAUSES and IMAGINE Study investigators recruited the patients and provided the patient ES and WGS data analyzed in this study. AM re-aligned a subset of the EGA GS data and implemented STRetch on a subset of the IMAGINE GS data. ED, ME, and IB provided critical inputs on the experimental design, manuscript structure, and its revision. ISRB drafted the manuscript. ISRB, JJP, RC, CL, ED, ME, IB, and $J M F$ revised the manuscript. The authors read and approved the final manuscript.

\section{Funding}

The IMAGINE Study is supported by the Canadian Institutes of Health Research (CIHR-SCA-145104) through CHILD-BRIGHT (Child Health Initiative Limiting Disability-Brain Research Improving Growth and Health Trajectories), with additional support provided by BC Children's Hospital Foundation and the Michael Smith Foundation for Health Research (MSFHR). The CAUSES Study is funded by Mining for Miracles, British Columbia Children's Hospital Foundation, and Genome British Columbia. Indhu Shree Rajan Babu is a recipient of the MSFHR Research Trainee Award. We thank the Rare Disease Foundation for funding our research on developing a bioinformatics pipeline to analyze STRs in nextgeneration sequencing data (grant \#2332).

\section{Availability of data and materials}

The PCR-free whole-genome sequencing data of Coriell samples with known short tandem repeat expansions analyzed in the current study can be accessed in the European Genome-phenome Archive repository: https:// www.ebi.ac.uk/ega/datasets/EGAD00001003562 [10]. Individual patient clinical or genomic data from the CAUSES [25] and IMAGINE [26] Studies are not available because they are potentially individually identifiable and not consented for public release. Investigators who wish access to the summary data of CAUSES and IMAGINE Studies can contact Dr. Jan Friedman (jmf@bcchr. ca). Results of all analyses based on the European Genome-phenome Archive, CAUSES, and IMAGINE datasets are included in this published article and its supplementary information files.

\section{Web resources}

lobSTR - https://github.com/mgymrek/lobstr-code

RepeatSeq - https://github.com/adaptivegenome/repeatseq

HipSTR - https://github.com/tfwillems/HipSTR

TREDPARSE - https://github.com/humanlongevity/tredparse

ExpansionHunter - https://github.com/llumina/ExpansionHunter

STRetch — https://github.com/Oshlack/STRetch

exSTRa - https://bahlolab.github.io/exSTRa/doc/exSTRa.html

GangSTR - https://github.com/gymreklab/GangSTR

Ambry IDT bed - https://www.idtdna.com/pages/products/next-generationsequencing/hybridization-capture/lockdown-panels/xgen-exome-researchpanel-v2

Centogene bed — https:/support.illumina.com/sequencing/sequencing_ kits/nextera-rapid-capture-exome-kit/downloads.html

GSC bed - https://earray.chem.agilent.com/suredesign/home.htm

ART — https://www.niehs.nih.gov/research/resources/software/biostatistics/ art/index.cfm

BWA — http://bio-bwa.sourceforge.net/

Isaac — https://github.com/llumina/Isaac4

scikit-learn - https://scikit-learn.org/stable/

MLxtend — http://rasbt.github.io/mlxtend/
European Genome-phenome Archive dataset — https://www.ebi.ac.uk/ega/ datasets/EGAD00001003562

Picard - https://broadinstitute.github.io/picard/

CAUSES Study - https://bcchr.ca/news/causes-research-clinic-children-andfamilies-undiagnosed-disorders

IMAGINE Study — https://www.child-bright.ca/imagine

GraphAlignmentViewer — https://github.com/llumina/

GraphAlignmentViewer

\section{Declarations}

\section{Ethics approval and consent to participate}

The clinical next-generation sequencing datasets used in this study are from patients and their biological parents recruited for the CAUSES or IMAGINE Study. Written informed consent was obtained from all CAUSES and IMAG INE Study participants, and both Studies were approved by the Institutional Review Board of the Children's and Women's Health Centre of British Columbia and the University of British Columbia (H15-00092 and H16-02126, respectively). The research in this study conforms to the principles of the Helsinki Declaration.

\section{Consent for publication}

Not applicable

\section{Competing interests}

ED and MAE are employees of Illumina, Inc., a public company that develops and markets systems for genetic analysis. The remaining authors declare that they have no competing interests.

\section{Author details}

${ }^{1}$ Department of Medical Genetics, University of British Columbia and Children's \& Women's Hospital, Vancouver, BC V6H3N1, Canada. 'Department of Medical and Molecular Genetics, King's College London, Strand, London WC2R 2LS, UK. ${ }^{3}$ Canada's Michael Smith Genome Sciences Centre, BC Cancer Agency, Vancouver, BC V5Z4S6, Canada. ${ }^{4}$ Bioinformatics Graduate Program, University of British Columbia, Vancouver, BC V6T1Z4, Canada. ${ }^{5}$ Illumina Inc., San Diego, CA 92121, USA.

\section{Received: 3 September 2020 Accepted: 5 July 2021}

Published online: 09 August 2021

\section{References}

1. Tang H, Kirkness EF, Lippert C, Biggs WH, Fabani M, Guzman E, et al. Profiling of short-tandem-repeat disease alleles in 12,632 human whole genomes. Am J Hum Genet. 2017 Nov;101(5):700-15. https://doi.org/10.101 6/j.ajhg.2017.09.013.

2. Sznajder $Ł J$, Swanson MS. Short tandem repeat expansions and RNAmediated pathogenesis in myotonic dystrophy. Int J Mol Sci. 2019 Jul;9: 20(13).

3. Paulson H. Repeat expansion diseases. Handb Clin Neurol. 2018;147:105-23. https://doi.org/10.1016/B978-0-444-63233-3.00009-9.

4. Salcedo-Arellano MJ, Dufour B, McLennan Y, Martinez-Cerdeno V, Hagerman $R$. Fragile $X$ syndrome and associated disorders: clinical aspects and pathology. Neurobiol Dis. 2020 Mar;136:104740. https://doi.org/10.1016/j. nbd.2020.104740.

5. Mila M, Alvarez-Mora MI, Madrigal I, Rodriguez-Revenga L. Fragile X syndrome: an overview and update of the FMR1 gene. Clin Genet. 2018 Feb;93(2):197-205. https://doi.org/10.1111/cge.13075.

6. Nelson DL, Orr HT, Warren ST. The unstable repeats--three evolving faces of neurological disease. Neuron. 2013 Mar 6;77(5):825-43. https://doi.org/10.1 016/j.neuron.2013.02.022.

7. Semaka A, Creighton S, Warby S, Hayden MR. Predictive testing for Huntington disease: interpretation and significance of intermediate alleles. Clin Genet. 2006 Oct;70(4):283-94. https://doi.org/10.1111/j.1399-0004.2006. 00668.x.

8. Usdin K, Grabczyk E. DNA repeat expansions and human disease. Cell Mol Life Sci CMLS. 2000 Jun;57(6):914-31. https://doi.org/10.1007/PL00000734.

9. Wallace $S E$, Bean $L$ J. Resources for genetics professionals — genetic disorders caused by nucleotide repeat expansions and contractions [internet]. Seattle: University of Washington; 2019. [cited 2021 Mar 31] Available from: https://www.ncbi.n/m.nih.gov/books/NBK535148/ 
10. Dolzhenko E, van Vugt JJFA, Shaw RJ, Bekritsky MA, van Blitterswijk M, Narzisi G, et al. Detection of long repeat expansions from PCR-free wholegenome sequence data. Genome Res. 2017 Nov;27(11):1895-903. https://doi.org/10.1101/gr.225672.117.

11. Dashnow H, Lek M, Phipson B, Halman A, Sadedin S, Lonsdale A, et al. STRetch: detecting and discovering pathogenic short tandem repeat expansions. Genome Biol. 8;19(1):121.

12. Tankard RM, Bennett MF, Degorski P, Delatycki MB, Lockhart PJ, Bahlo M. Detecting expansions of tandem repeats in cohorts sequenced with shortread sequencing data. Am J Hum Genet. 2018 Dec;103(6):858-73. https://doi.org/10.1016/j.ajhg.2018.10.015.

13. Dolzhenko E, Deshpande V, Schlesinger F, Krusche P, Petrovski R, Chen S, et al. ExpansionHunter: a sequence-graph based tool to analyze variation in short tandem repeat regions. Bioinformatics [Internet]. 2019 May; Available from: https://www.ncbi.n/m.nih.gov/pubmed/31134279

14. Mousavi N, Shleizer-Burko S, Yanicky R, Gymrek M. Profiling the genomewide landscape of tandem repeat expansions. Nucleic Acids Res [Internet]. 2019 Jun; Available from: https://www.ncbi.nlm.nih.gov/pubmed/31194863

15. Gymrek M, Golan D, Rosset S, Erlich Y. lobSTR: a short tandem repeat profiler for personal genomes. Genome Res. 2012 Jun;22(6):1154-62. https://doi.org/10.1101/gr.135780.111.

16. Willems T, Zielinski D, Yuan J, Gordon A, Gymrek M, Erlich Y. Genome-wide profiling of heritable and de novo STR variations. Nat Methods. 2017 Jun; 14(6):590-2. https://doi.org/10.1038/nmeth.4267.

17. Highnam G, Franck C, Martin A, Stephens C, Puthige A, Mittelman D. Accurate human microsatellite genotypes from high-throughput resequencing data using informed error profiles. Nucleic Acids Res. 2013 Jan;41(1):e32. https://doi.org/10.1093/nar/gks981.

18. Langmead B, Salzberg SL. Fast gapped-read alignment with bowtie 2. Nat Methods. 2012 Mar;9(4):357-9. https://doi.org/10.1038/nmeth.1923.

19. Halman A, Oshlack A. Accuracy of short tandem repeats genotyping tools in whole exome sequencing data. F1000Research. 2020;9:200. https://doi.org/1 0.12688/f1000research.22639.1.

20. Rocca MS, Ferrarini M, Msaki A, Vinanzi C, Ghezzi M, De Rocco Ponce M, et al. Comparison of NGS panel and Sanger sequencing for genotyping CAG repeats in the AR gene. Mol Genet Genomic Med. 2020;8(6):e1207. https://doi.org/10.1002/mgg3.1207.

21. Raczy C, Petrovski R, Saunders CT, Chorny I, Kruglyak S, Margulies EH, et al. Isaac: ultra-fast whole-genome secondary analysis on Illumina sequencing platforms. Bioinformatics. 2013 Aug;29(16):2041-3. https://doi.org/10.1093/ bioinformatics/btt314.

22. Li H, Durbin R. Fast and accurate short read alignment with burrowswheeler transform. Bioinformatics. 2009 Jul 15;25(14):1754-60. https://doi. org/10.1093/bioinformatics/btp324.

23. Lee $H$, Lee K-W, Lee T, Park D, Chung J, Lee C, et al. Performance evaluation method for read mapping tool in clinical panel sequencing. Genes Genomics. 2018 Feb 1;40(2):189-97. https://doi.org/10.1007/s13258-0170621-9.

24. Huang W, Li L, Myers JR, Marth GT. ART: a next-generation sequencing read simulator. Bioinformatics. 2012 Feb 15;28(4):593-4. https://doi.org/10.1093/ bioinformatics/btr708.

25. Elliott AM, du Souich C, Adam S, Dragojlovic N, van Karnebeek C, Nelson $\mathrm{TN}$, et al. The genomic consultation service: a clinical service designed to improve patient selection for genome-wide sequencing in British Columbia. Mol Genet Genomic Med. 2018;6(4):592-600. https://doi.org/10.1002/ mgg3.410.

26. Diagnosis Using Integrated Metabolomics And Genomics In Neurodevelopment (IMAGINe) [Internet]. CHILD-BRIGHT Network. [cited 2021 Jul 1]. Available from: https://www.child-bright.ca/imagine

27. Picard tools - by Broad Institute [Internet]. [cited 2021 Jun 14]. Available from: http://broadinstitute.github.io/picard/

28. Krzywinski M, Altman N. Classification and regression trees. Nat Methods. 2017 Aug 1;14(8):757-8. https://doi.org/10.1038/nmeth.4370.

29. Pedregosa F, Varoquaux G, Gramfort A, Michel V, Thirion B, Grisel O, et al. Scikit-learn: machine learning in python. J Mach Learn Res. 2011;12(85): 2825-30.

30. Raschka S. MLxtend: Providing machine learning and data science utilities and extensions to Python's scientific computing stack. J Open Source Softw. 2018;3(24):638. https://doi.org/10.21105/joss.00638.

31. Illumina/GraphAlignmentViewer [Internet]. Illumina; 2020 [cited 2021 Mar 31]. Available from: https://github.com/llumina/GraphAlignmentViewer
32. van Kuilenburg ABP, Tarailo-Graovac M, Richmond PA, Drögemöller BI, Pouladi MA, Leen R, et al. Glutaminase deficiency caused by short tandem repeat expansion in. N Engl J Med. 380(15):1433-41.

33. Sone J, Mitsuhashi S, Fujita A, Mizuguchi T, Hamanaka K, Mori K, et al. Longread sequencing identifies GGC repeat expansions in NOTCH2NLC associated with neuronal intranuclear inclusion disease. Nat Genet. 2019 Aug;51(8):1215-21. https://doi.org/10.1038/s41588-019-0459-y.

34. Tian $Y$, Wang $J$, Huang $W$, Zeng $S$, Jiao B, Liu Z, et al. Expansion of humanspecific GGC repeat in neuronal intranuclear inclusion disease-related disorders. Am J Hum Genet. 2019 Jul;105(1):166-76. https://doi.org/10.1016/ j.ajhg.2019.05.013.

35. Florian RT, Kraft F, Leitão E, Kaya S, Klebe S, Magnin E, et al. Unstable TTTTA TTTCA expansions in MARCH6 are associated with familial adult myoclonic epilepsy type 3. Nat Commun. 2019 Oct 29;10(1):4919. https://doi.org/10.103 8/s41467-019-12763-9.

36. Corbett MA, Kroes T, Veneziano L, Bennett MF, Florian R, Schneider AL, et al. Intronic ATTTC repeat expansions in STARD7 in familial adult myoclonic epilepsy linked to chromosome 2. Nat Commun. 2019 Oct 29;10(1):4920. https://doi.org/10.1038/s41467-019-12671-y.

37. Lee SE, Sias AC, Mandelli ML, Brown JA, Brown AB, Khazenzon AM, et al. Network degeneration and dysfunction in presymptomatic C9ORF72 expansion carriers. Neurolmage Clin. 2017;14:286-97. https://doi.org/10.101 6/j.nicl.2016.12.006.

38. Le Blanc G, Jetté Pomerleau V, McCarthy J, Borroni B, van Swieten J, Galimberti D, et al. Faster cortical thinning and surface area loss in presymptomatic and symptomatic C9orf72 repeat expansion adult carriers. Ann Neurol. 2020 Jul;88(1):113-22. https://doi.org/10.1002/ana.25748.

39. Chu Y, Hu J, Liang H, Kanchwala M, Xing C, Beebe W, et al. Analyzing presymptomatic tissue to gain insights into the molecular and mechanistic origins of late-onset degenerative trinucleotide repeat disease. Nucleic Acids Res. 2020 Jul 9;48(12):6740-58. https://doi.org/10.1093/nar/gkaa422.

40. DeKosky ST, Marek K. Looking backward to move forward: early detection of neurodegenerative disorders. Science. 2003 Oct 31;302(5646):830-4. https:// doi.org/10.1126/science.1090349.

41. Mantere $T$, Kersten $S$, Hoischen A. Long-read sequencing emerging in medical genetics. Front Genet. 2019;10:426. https://doi.org/10.3389/fgene.2 019.00426.

42. Hunter JE, Berry-Kravis E, Hipp H, Todd PK. FMR1 Disorders. In: Adam MP, Ardinger $\mathrm{HH}$, Pagon RA, Wallace SE, Bean L, Mirzaa G, et al., editors. GeneReviews ${ }^{\circledast}$ [Internet]. Seattle (WA): University of Washington, Seattle; 1993 [cited 2021 Mar 31]. Available from: http://www.ncbi.nlm.nih.gov/ books/NBK1384/

43. Bird TD. Myotonic Dystrophy Type 1. GeneReviews ${ }^{\circledast}$ [Internet]. Seattle (WA): University of Washington, Seattle; 1993-2018. Available from: https://www. ncbi.nlm.nih.gov/books/NBK1165/; 1999.

44. Raz O, Biezuner T, Spiro A, Amir S, Milo L, Titelman A, et al. Short tandem repeat stutter model inferred from direct measurement of in vitro stutter noise. Nucleic Acids Res. 2019 Mar 18;47(5):2436-45. https://doi.org/10.1093/ nar/gky1318.

\section{Publisher's Note}

Springer Nature remains neutral with regard to jurisdictional claims in published maps and institutional affiliations. 